\title{
Adaptive Resource Allocation for Improved DF Aided Downlink Multi-user OFDM Systems
}

\author{
Yong Liu, and Wen Chen, Senior member, IEEE
}

\begin{abstract}
In this letter, we propose a joint resource allocation algorithm for an OFDM-based multi-user system assisted by an improved Decode-and-Forward (DF) relay. We aim at maximizing the sum rate of the system by jointly optimizing subcarrier pairing, subcarrier pair-user assignment, and power allocation in such a single DF relay system. When the relay does not perform any transmission on some subcarriers in the second phase, we further allow the source to transmit new symbols on these inactive subcarriers. We effectively solve the formulated mixed integer programming problem by using continuous relaxation and dual minimization methods. Numerical results verify the theoretical analysis, and illustrate the remarkable gains resulted from the extra direct-link transmissions.
\end{abstract}

Index Terms-OFDM, Multi-user, Subcarrier Pairing, Power Allocation, Decode-and-Forward.

\section{INTRODUCTION}

$\mathbf{P}$ OWER allocation and subcarrier pairing have attracted much research attention in OFDM-based relaying systems [1]-[12], due to the limited budget of power, and independent fading on subcarriers in each hop. Most of the previous works focus on single-user scenarios. Only a few literatures consider about multi-user cases [7]-[10]. Authors in [7] investigate the problems of subcarrier allocation, subcarrier pairing and power allocation in a relay aided two-hop uplink multi-user transmission model. Authors in [8] work on the similar problem but for multi-relay channels. Authors in [9] also study the resource allocation for the multi-user multirelay model, and the optimal subcarrier allocation with QoS constraint is solved by using a graph theoretical approach. On the other hand, the joint resource allocation for relay aided downlink multi-destination networks is considered in [10][12]. However, in these works, the source always keeps silent during the second phase, irrespective to whether the relay subcarriers are active or not. In this paper, we consider an improved DF relaying scheme. For the circumstances where the relay does not forward on some subcarriers because doing so does not improve the sum rate, we further allow the source to transmit extra symbols on these idle subcarriers in the source-destination link in the second phase. Authors in [13] and [14] consider a similar transmission scheme, but for single-user scenario. Jointly optimizing subcarrier pairing,

Manuscript received May 21, 2012; revised July 18, 2012, and accepted July 30,2012 . The associate editor coordinating the review of this paper and approving it for publication was Dusit Niyato.

Yong Liu and Wen Chen are with the Department of Electronic Engineering, Shanghai Jiao Tong University, Shanghai, 200240 PRC. e-mail: \{yongliu1982, wenchen\}@ sjtu.edu.cn

This work is supported by national 973 project \#2012CB316106 and \#2009CB824904, by NSF China \#60972031 and \#61161130529.

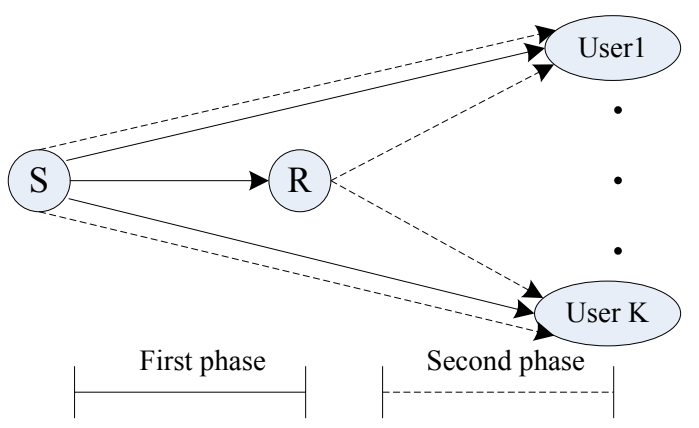

Fig. 1. OFDM based improved DF diversity downlink multi-user model.

subcarrier pair-user assignment and power allocation for multiuser channels makes the problem more general and difficult.

\section{SYSTEM DESCRIPTION}

The improved OFDM DF diversity model is shown in Fig. 1, in which a source communicates with $K$ users assisted by a single relay with $N$ subcarriers. The channel gains are assumed to be constant during two phases. In the first phase, the source broadcasts its signal while the relay and the destination listen. In the second phase, the relay transmits an encoded version of the received signal on some subcarriers, while the source transmits a new modulated symbol on the subcarriers that are not used by the relay. We use $\operatorname{SP}(k, m n)$ to denote the subcarrier $m$ in the first phase pairing with the subcarrier $n$ in the second phase, and the pair of channels $(m, n)$ is assigned to user $k$. We respectively denote by $h_{S D_{k}}^{m}, h_{S R}^{m}$ and $h_{R D_{k}}^{n}$ the channel coefficients of source-user $k$, source-relay, and relayuser $k$ on the corresponding subcarriers. If the relay keeps silent on subcarrier $n$ during the second phase, the channel coefficient of the extra source-destination link is denoted by $h_{S D_{k}}^{n}$. Then the corresponding normalized channel gains are respectively $\gamma_{S, D_{k}}^{m}=\left|h_{S D_{k}}^{m}\right|^{2} / \sigma_{k}^{2}, \gamma_{S, R}^{m}=\left|h_{S R}^{m}\right|^{2} / \sigma_{r}^{2}$, $\gamma_{R, D_{k}}^{n}=\left|h_{R D_{k}}^{n}\right|^{2} / \sigma_{k}^{2}$ and $\gamma_{S, D_{k}}^{n}=\left|h_{S D_{k}}^{n}\right|^{2} / \sigma_{k}^{2}$, in which $\sigma_{k}^{2}$ and $\sigma_{r}^{2}$ are noise powers at the user $k$ and the relay.

Given a potential subcarrier user pair $\operatorname{SP}(k, m n)$ in the improved DF diversity model, its achievable rate is given in (1), where $P_{S, D_{k}}^{m}$ and $P_{S, D_{k}}^{n}$ denote the powers used by the direct-link of $\mathrm{SP}(k, m n)$ in the first and second phases, respectively. $P_{S}^{m}$ and $P_{R, D_{k}}^{n}$ respectively denote the source and relay powers in the relaying mode.

Define a subcarrier pairing parameter $t_{m, n} \in\{0,1\}$, whose value is 1 if subcarriers $m$ and $n$ are paired, and 0 otherwise. Denote $\pi_{k, m n}$ as the user assignment parameter, whose value is 1 if subcarrier pair $(m, n)$ is assigned to user $k$, and 0 


$$
R^{k, m n}= \begin{cases}\frac{1}{2} \log _{2}\left(1+\gamma_{S, D_{k}}^{m} P_{S, D_{k}}^{m}\right)+\frac{1}{2} \log _{2}\left(1+\gamma_{S, D_{k}}^{n} P_{S, D_{k}}^{n}\right), & \text { idle mode, } \\ \frac{1}{2} \min \left\{\log _{2}\left(1+P_{S}^{m} \gamma_{S, D_{k}}^{m}+P_{R, D_{k}}^{n} \gamma_{R, D_{k}}^{n}\right), \log _{2}\left(1+P_{S}^{m} \gamma_{S R}^{m}\right)\right\}, & \text { relaying mode. }\end{cases}
$$

otherwise. Since the condition for using the relay depends not only on the channel gains but also on the power allocation, we further denote $\varphi_{k, m n} \in\{0,1\}$ to show whether the relay is used, i.e., the relay is used for $\operatorname{SP}(k, m n)$ if $\varphi_{k, m n}=1$. Otherwise, it is not used. We aim to maximize the sum rate by jointly optimizing subcarrier pairing, subcarrier pair-user assignment, and power allocation under total power constraint.

\section{Joint Resource Allocation}

\section{A. Optimization Problem Formulation}

Given a potential subcarrier pair $\operatorname{SP}(k, m n)$ working in the relaying mode, the achievable rate in (1) is maximized when

$$
\log _{2}\left(1+P_{S}^{m} \gamma_{S R}^{m}\right)=\log _{2}\left(1+P_{S}^{m} \gamma_{S, D_{k}}^{m}+P_{R, D_{k}}^{n} \gamma_{R, D_{k}}^{n}\right)
$$

that is,

$$
P_{S}^{m} \gamma_{S R}^{m}=P_{S}^{m} \gamma_{S, D_{k}}^{m}+P_{R, D_{k}}^{n} \gamma_{R, D_{k}}^{n}
$$

Let $P^{k, m n}=P_{S}^{m}+P_{R, D_{k}}^{n}$ for $\operatorname{SP}(k, m n)$, we can express the achievable rate of $\operatorname{SP}(k, m n)$ working in the relaying mode as

$$
R_{R}^{k, m n}=\frac{1}{2} \log _{2}\left(1+\gamma^{k, m n} P^{k, m n}\right),
$$

by letting

$$
\left\{\begin{array}{l}
P_{S}^{m}=\frac{\gamma_{R, D_{k}}^{n}}{\gamma_{R, D_{k}}^{n}+\gamma_{S R}^{m}-\gamma_{S, D_{k}}^{m}} P^{k, m n}, \\
P_{R, D_{k}}^{n}=\frac{\gamma_{S R}^{m}-\gamma_{S, D_{k}}^{m}}{\gamma_{R, D_{k}}^{n}+\gamma_{S R}^{m}-\gamma_{S, D_{k}}^{m}} P^{k, m n}, \\
\gamma^{k, m n}=\frac{\gamma_{S R}^{m} \gamma_{R, D_{k}}^{n}}{\gamma_{R, D_{k}}^{n}+\gamma_{S R}^{m}-\gamma_{S, D_{k}}^{m}} .
\end{array}\right.
$$

Then the total end-to-end sum rate can be expressed as

$$
\begin{array}{r}
\mathbf{R}=\sum_{k=1}^{K} \sum_{m=1}^{N} \sum_{n=1}^{N} \frac{t_{m, n}}{2} \pi_{k, m n}\left\{\varphi_{k, m n} \log _{2}\left(1+\gamma^{k, m n} P^{k, m n}\right)\right. \\
+\left(1-\varphi_{k, m n}\right)\left[\log _{2}\left(1+\gamma_{S, D_{k}}^{m} P_{S, D_{k}}^{m}\right)\right. \\
\left.\left.+\log _{2}\left(1+\gamma_{S, D_{k}}^{n} P_{S, D_{k}}^{n}\right)\right]\right\}
\end{array}
$$

and the sum rate optimization can be formulated as

$$
\begin{aligned}
& \underset{\{\mathbf{t}, \boldsymbol{\pi}, \boldsymbol{\varphi}, \mathbf{P}\}}{\max } \mathbf{R} \\
& \text { s.t. } \mathbf{C} 1: \sum_{m=1}^{N} t_{m, n}=1, \forall n, \quad \mathbf{C} 2: \sum_{n=1}^{N} t_{m, n}=1, \forall m, \\
& \mathbf{C} 3: \sum_{m=1}^{N} \sum_{n=1}^{N} \sum_{k=1}^{K} t_{m, n} \pi_{k, m n} \varphi_{k, m n} P^{k, m n}+ \\
& \quad \mathbf{C} 4: \sum_{k=1}^{K} \pi_{k, m n}=1, \forall m, n, \mathbf{C} 5: P^{k, m n}, P_{S, D_{k}}^{m}, P_{S, D_{k}}^{n} \geq 0, \\
& \mathbf{C} 6: t_{m, n}, \pi_{k, m n}, \varphi_{k, m n} \in\{0,1\},
\end{aligned}
$$

where $\mathbf{P}=\left(P^{k, m n}, P_{S, D_{k}}^{m}, P_{S, D_{k}}^{n}\right) \in\left(\mathbf{R}^{3}\right)^{N \times N}, \mathbf{t}=$ $\left(t_{m, n}\right) \in \mathbf{R}^{N \times N}, \boldsymbol{\pi}$ and $\boldsymbol{\varphi}$ are matrices with entries $\pi_{k, m n}$ and $\varphi_{k, m n}$ respectively. $\mathbf{C} 1$ and $\mathbf{C} 2$ correspond to the pairing constraint that each subcarrier $m$ in the first phase only pairs with one subcarrier $n$ in the second phase. C3 denotes the total power constraint, in which $P_{t}$ denotes the sum of transmit powers at all the nodes. $\mathbf{C} 4$ guarantees that each subcarrier pair can only be assigned to one user.

Since the sum rate optimization mentioned above is a mixed integer programming problem, which is hard to solve, we relax the integer constraint $\mathbf{C} 6: t_{m, n}, \pi_{k, m n}, \varphi_{k, m n} \in\{0,1\}$ as C7 : $t_{m, n}, \pi_{k, m n}, \varphi_{k, m n} \in[0,1]$ as in [12],[15]. Then the rate optimization problem can be expressed as (7) with a new power constraint C8: $\sum_{m=1}^{N} \sum_{n=1}^{N} \sum_{k=1}^{K}\left(S^{k, m n}+\right.$ $\left.S_{S, D_{k}}^{m}+S_{S, D_{k}}^{n}\right) \leq P_{t}$, where we introduce new variables $S^{k, m n}=t_{m, n} \pi_{k, m n} \varphi_{k, m n} P^{k, m n}, S_{S, D_{k}}^{m}=t_{m, n} \pi_{k, m n}(1-$ $\left.\varphi_{k, m n}\right) P_{S, D_{k}}^{m}$ and $S_{S, D_{k}}^{n}=t_{m, n} \pi_{k, m n}\left(1-\varphi_{k, m n}\right) P_{S, D_{k}}^{n}$ to denote the actual powers consumed by the relaying mode and idle mode, respectively. To guarantee $t_{m, n}, \pi_{k, m n}$ and $\varphi_{k, m n}$ being integer-valued, we solve the problem by the dual method [15]. Since the objective function of (7) is composed of three concave functions, (7) is thus a standard convex programming problem. Therefore, the optimization problem satisfies the time-sharing condition that guarantees zero duality gap [15].

\section{B. Optimal Solution in Dual Domain}

Construct the Lagrange function for (7) as

$$
\begin{aligned}
& L(\mathbf{t}, \boldsymbol{\pi}, \boldsymbol{\varphi}, \mathbf{S}, \lambda)=\tilde{\mathbf{R}}- \\
& \quad \lambda\left(\sum_{m=1}^{N} \sum_{n=1}^{N} \sum_{k=1}^{K}\left(S^{k, m n}+S_{S, D_{k}}^{m}+S_{S, D_{k}}^{n}\right)-P_{t}\right),
\end{aligned}
$$

where $\tilde{\mathbf{R}}$ denotes the optimization objective in (7), and $\lambda$ is the dual variable corresponding to $\mathbf{C} 8$. Then the dual objective function is

$$
g(\lambda)=\max _{\{\mathbf{S}, \mathbf{t}, \boldsymbol{\pi}, \boldsymbol{\varphi}\}} L(\mathbf{t}, \boldsymbol{\pi}, \boldsymbol{\varphi}, \mathbf{S}, \lambda), \text { s.t. } \mathbf{C} 1, \mathbf{C} 2, \mathbf{C} 4, \mathbf{C} 7,
$$

and the dual problem is

$$
\min _{\{\lambda\}} g(\lambda) \quad \text { s.t. } \lambda \geq 0 .
$$

A dual function is always optimized by first optimizing some variables and then optimizing the others. We first take derivatives of (8) with respect to $S^{k, m n}, S_{S, D_{k}}^{m}$, and $S_{S, D_{k}}^{n}$ and obtain

$$
\begin{aligned}
& S_{*}^{k, m n}=t_{m, n} \pi_{k, m n} \varphi_{k, m n}\left[\frac{1}{2 \lambda}-\frac{1}{\gamma^{k, m n}}\right]^{+}, \\
& S_{S, D_{k}}^{m *}=t_{m, n} \pi_{k, m n}\left(1-\varphi_{k, m n}\right)\left[\frac{1}{2 \lambda}-\frac{1}{\gamma_{S, D_{k}}^{m}}\right]^{+}, \\
& S_{S, D_{k}}^{n *}=t_{m, n} \pi_{k, m n}\left(1-\varphi_{k, m n}\right)\left[\frac{1}{2 \lambda}-\frac{1}{\gamma_{S, D_{k}}^{n}}\right]^{+},
\end{aligned}
$$




$$
\begin{gathered}
\max _{\{\mathbf{t}, \boldsymbol{\pi}, \boldsymbol{\varphi}, \mathbf{S}\}} \sum_{k=1}^{K} \sum_{m=1}^{N} \sum_{n=1}^{N} \frac{t_{m, n}}{2} \pi_{k, m n}\left\{\varphi_{k, m n} \log _{2}\left(1+\gamma^{k, m n} \frac{S^{k, m n}}{t_{m, n} \pi_{k, m n} \varphi_{k, m n}}\right)+\left(1-\varphi_{k, m n}\right) .\right. \\
\left.\left[\log _{2}\left(1+\gamma_{S, D_{k}}^{m} \frac{S_{S, D_{k}}^{m}}{t_{m, n} \pi_{k, m n}\left(1-\varphi_{k, m n}\right)}\right)+\log _{2}\left(1+\gamma_{S, D_{k}}^{n} \frac{S_{S, D_{k}}^{n}}{t_{m, n} \pi_{k, m n}\left(1-\varphi_{k, m n}\right)}\right)\right]\right\},
\end{gathered}
$$

in which $[x]^{+}=\max \{0, x\}$.

If we denote the rate contribution of $\operatorname{SP}(k, m n)$ to the Lagrange function (8) in the relaying mode and idle mode as $R_{m, n}^{R}$ and $R_{m, n}^{I}$ respectively, then they can be obtained by substituting (11) into (8), that is

$$
\begin{aligned}
R_{m, n}^{R}= & \frac{1}{2} \log \left(1+\gamma^{k, m n} \tilde{S}_{*}^{k, m n}\right)-\lambda \tilde{S}_{*}^{k, m n}, \\
R_{m, n}^{I}= & \frac{1}{2} \log \left\{\left(1+\gamma_{S, D_{k}}^{m} \tilde{S}_{S, D_{k}}^{m *}\right)\left(1+\gamma_{S, D_{k}}^{n} \tilde{S}_{S, D_{k}}^{n *}\right)\right\} \\
& -\lambda\left(\tilde{S}_{S, D_{k}}^{m *}+\tilde{S}_{S, D_{k}}^{n *}\right),
\end{aligned}
$$

in which we denote $\tilde{S}_{*}^{k, m n}=\left[\frac{1}{2 \lambda}-\frac{1}{\gamma^{k, m n}}\right]^{+}, \tilde{S}_{S, D_{k}}^{m *}=$ $\left[\frac{1}{2 \lambda}-\frac{1}{\gamma_{S, D_{k}}^{m}}\right]^{+}$and $\tilde{S}_{S, D_{k}}^{n *}=\left[\frac{1}{2 \lambda}-\frac{1}{\gamma_{S, D_{k}}^{n}}\right]^{+}$for briefness. Easily we obtain the optimal solution for $\varphi_{k, m n}$ as

$$
\varphi_{k, m n}^{*}= \begin{cases}1, & \text { when } R_{m, n}^{R}>R_{m, n}^{I}, \\ 0, & \text { otherwise. }\end{cases}
$$

The optimal $\varphi_{k, m n}^{*}$ tells us whether it is better to use relay for the subcarrier-user pair $\operatorname{SP}(k, m n)$. Substitute (11), 13) and (12) into (8), the original Lagrange function (8) can be simplified as

$$
L(\mathbf{t}, \boldsymbol{\pi}, \lambda)=t_{m, n} \pi_{k, m n} \Pi_{k, m n}+\lambda P_{t},
$$

where $\Pi_{k, m n}=\varphi_{k, m n}^{*} R_{m, n}^{R}+\left(1-\varphi_{k, m n}^{*}\right) R_{m, n}^{I}$. We can observe from (14) that, to maximize $L(\mathbf{t}, \boldsymbol{\pi}, \lambda)$, the subcarrier pair $(m, n)$ should be assigned to the user with maximum value of $\Pi_{k, m n}$, that is,

$$
\pi_{k, m n}^{*}=\left\{\begin{array}{l}
1: k=\arg \max _{k \in[1, ., K]} \Pi_{k, m n} \\
0: \text { otherwise. }
\end{array}\right.
$$

If we denote $\Pi_{m n}=\Pi_{k, m n}^{*}=\max _{k \in[1, ., K]} \Pi_{k, m n}$, the dual objective function (9) can be further simplified as the following linear optimization problem

$$
\begin{aligned}
& g(\lambda)=\max _{\{\mathbf{t}\}} \sum_{k=1}^{K} \sum_{m=1}^{N} \sum_{n=1}^{N}\left\{t_{m, n} \Pi_{m n}+\lambda P_{t}\right\}, \\
& \text { s.t. } \quad \mathbf{C} 1, \mathbf{C} 2, \mathbf{C} 7,
\end{aligned}
$$

which is well known as the two-dimensional assignment problem [16]. The Hungarian Algorithm [16] is an efficient algorithm to solve such assignment problem with complexity that is a polynomial function in $N$. Without loss of generality, we can express the assignment result as

$$
t_{m, n}^{*}=\left\{\begin{array}{l}
1:(m, n)=H u n g\left(\Pi_{m n}\right), \\
0: \text { otherwise. }
\end{array}\right.
$$

Finally, minimizing the dual function (10) is required according to the standard Lagrange dual method, which can be solved with the subgradient method [15], that is,

$$
\begin{aligned}
\lambda^{(i+1)}= & \lambda^{(i)}-a^{(i)}\left(P_{t}-\right. \\
& \left.\sum_{m=1}^{N} \sum_{n=1}^{N} \sum_{k=1}^{K}\left(S^{k, m n}+S_{S, D_{k}}^{m}+S_{S, D_{k}}^{n}\right)\right),
\end{aligned}
$$

where $i$ is the iteration number, $a$ is step size. With the updated $\lambda^{(i+1)}$ in each iteration, we can respectively obtain the new power allocation vectors, $\varphi_{k, m n}$ and $\pi_{k, m n}$ by (11), (13) and (15), and then update $t_{m, n}$ with the Hungarian Algorithm. The whole procedure can be described as in Algorithm 1.

Algorithm 1 The Proposed Resource Allocation Algorithm

Step 1: Initialize $\lambda, \max _{i t e r}$ and set $i=1$,

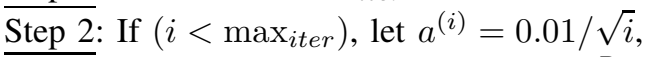

Step 3: Compute the rate contributions $R_{m, n}^{R}$ and $R_{m, n}^{I}$ by

(12) with $\lambda=\lambda^{(i)}$, and corresponding channel gains,

Step 4: Compute $\varphi_{k, m n}^{*(i)}$ by (13) using $R_{m, n}^{I}=R_{m, n}^{I(i)}$ and $R_{m, n}^{R}=R_{m, n}^{R(i)}$

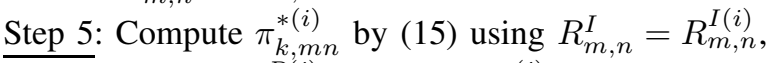
$R_{m, n}^{R}=R_{m, n}^{R(i)}, \varphi_{k, m n}^{*}=\varphi_{k, m n}^{*(i)}$ and $\lambda=\lambda^{(i)}$,

Step 6: Compute $t_{m, n}^{*}$ by (17) using $R_{m, n}^{I}=R_{m, n}^{I(i)}$, $R_{m, n}^{R}=R_{m, n}^{R(i)}, \varphi_{k, m n}^{*}=\varphi_{k, m n}^{*(i)}$ and $\pi_{k, m n}^{*}=\pi_{k, m n}^{*(i)}$,

Step 7: Compute $S_{*}^{k, m n}, S_{S, D_{k}}^{m *}$ and $S_{S, D_{k}}^{n *}$ by (11) using $t_{m, n}=t_{m, n}^{(i)}, \pi_{k, m n}^{*}=\pi_{k, m n}^{*(i)}, \varphi_{k, m n}^{*}=\varphi_{k, m n}^{*(i)}$ and $\lambda=\lambda^{(i)}$,

Step 8: Compute $\lambda^{(i+1)}$ by (18) using $\lambda^{(i)}, a^{(i)}$, $S_{*}^{k, m n}=S_{*(i)}^{k, m n}, S_{S, D_{k}}^{m *}=S_{S, D_{k}}^{m *(i)}, S_{S, D_{k}}^{n *}=S_{S, D_{k}}^{n *(i)}$,

Step 9: If $\frac{\left|\lambda^{(i+1)}-\lambda^{(i)}\right|}{\left|\lambda^{(i+1)}\right|}<\varepsilon$, exit and output the optimal solutions, otherwise set $i=i+1$ and go to Step 2 .

The complexity of each iteration in the proposed algorithm is almost dominated by the Hungarian Algorithm, which is $O\left(N^{3}\right)$ [16]. On the other hand, the complexity of exhaustive searching method is $O\left(N ! K^{N} 2^{N}\right)$. Obviously, the complexity of the proposed method is acceptable.

\section{Simulation Results}

We consider an OFDMA system with $K=4$, and each subcarriers channel experiencing flat-fading. We further assume $\sigma_{r}^{2}=\sigma_{d}^{2}$ for simplicity. The step size $a^{(i)}$ for the subgradient method is set to be $\frac{0.01}{\sqrt{i}}$. The source and relay are assumed to be placed on a horizontal line, and $d_{S R}=10$. The four users are uniformly placed on the right semicircle centered at the relay, and with radius $d_{R D}=5$. 


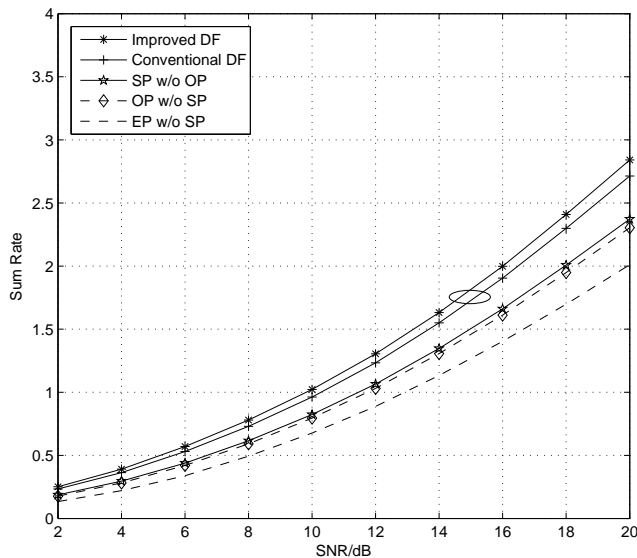

Fig. 2. Sum rate versus SNR for $N=4$, and $K=4$.

Fig. 2 is obtained by comparing the proposed algorithm with several schemes: equal power allocation without subcarrier pairing $(E P$ w/o $S P)$, optimal power allocation without subcarrier pairing (OPA w/o $S P$ ), equal power allocation with subcarrier pairing (EP with $S P$ ) and the joint power allocation and subcarrier pairing for conventional DF that does not use the idle subcarriers (Conventional DF). We find that the proposed algorithm as well as the Conventional DF always outperform the others, and the performance gaps increase as the SNR increases, which implies that the power allocation and subcarrier pairing indeed provides a substantial improvement to the system performance. On the other hand, the performance of improved relay protocol is superior to that of conventional DF, which results from the extra direct-link transmission in the second phase. For $S N R=4,10,18 \mathrm{~dB}$, the sum rate improvement percentage is $7.44 \%, 6.24 \% 4.7 \%$, respectively. We also find that the iterative algorithm quickly converges (e.g., 9 iterations).

The sum rates of the OFDMA system versus $N$ for the conventional DF and the improved DF modes are illustrated in Fig. 3. For $N=4,8,16,32,64$ respectively. 10000 of such two-phase periods are simulated. We observe that the rate gain of improved DF increases with $N$, which results from more frequency diversity and pairing flexibility of large $N$.

\section{CONCLUSion}

In this letter, we have proposed a joint resource allocation algorithm for the OFDM improved DF diversity channels, where the source is allowed to transmit new messages on the idle subcarriers that are not used in the second phase. With total power constraint, the joint optimization has been formulated as a mixed integer programming problem. We have solved this problem efficiently with polynomial complexity with the dual method. Simulation results have verified that a remarkable rate gain can be achieved with the extra direct-link transmissions.

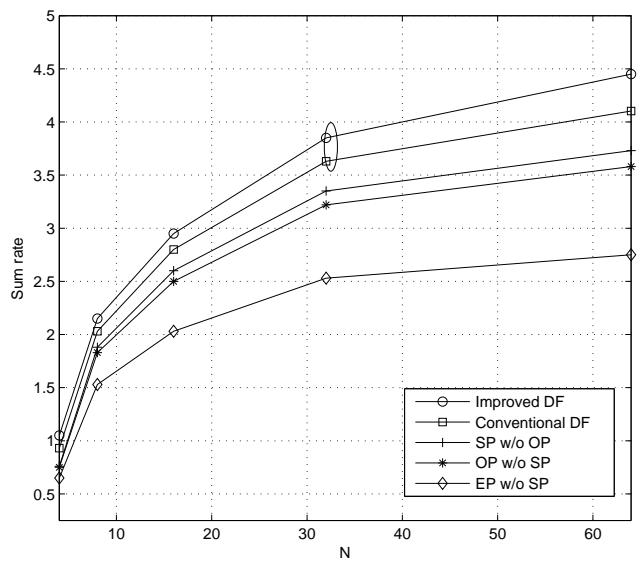

Fig. 3. Sum rate versus $N$ for $P_{t}=10$ and $K=4$.

\section{REFERENCES}

[1] M. Herdin, "A chunk based OFDM amplify-and-forward relaying scheme for 4G mobile radio systems," in Proc. IEEE Int. Conf. Communications (ICC), Jun. 2006.

[2] Y. Cui, V. Lau, and R. Wang, "Distributive subband allocation, power and rate control for relay-assisted OFDMA cellular system with imperfect system state knowledge," IEEE Trans. Wireless Commun., vol. 8, no. 10, pp. 5096-5102, Oct. 2009.

[3] H. Wan, W. Chen, and J. Ji, "Efficient Linear Transmission Strategy for MIMO Relaying Broadcast Channels with Direct Links," IEEE Wireless Communications Letters, vol. 1, no. 1, pp. 14-17, 2012.

[4] Z. Wang, W. Chen, and J. Li, "Efficient Beamforming for MIMO Relaying Broadcast Channel with Imperfect Channel Estimation," IEEE Transactions on Vehicular Technology, vol. 61, no. 1, pp. 419-426, 2012.

[5] H. Wan, and W. Chen, "Joint Source and Relay Design for Multiuser MIMO Non-regenerative Relay Networks with Direct Links," to be published in IEEE Transactions on Vehicular Technology, 2012.

[6] G. Sidhu, F. Gao, W. Chen, and W. Wang, "Joint Subcarrier Pairing and Power Loading in Relay Aided Cognitive Radio Networks," IEEE Wire. Commu. and Network. Conf. (WCNC2012), Apr. 2012.

[7] G. A. Sidhu, F. Gao, "Resource Allocation for Relay Aided Uplink Multiuser OFDMA System," in Proc. IEEE Conf. on WCNC, pp. 1-5, Apr. 2010.

[8] H. Jeong, J. H. Lee, H. Seo, "Resource Allocation for Uplink Multiuser OFDM Relay Networks with Fairness Constraints," in Proc. IEEE Conf. on VTC Spring, pp. 1-5, Apr. 2009.

[9] G. Li, H. Liu, "Resource Allocation for OFDMA Relay Networks With Fairness Constraints," IEEE J. Sel. Areas Commun., vol. 24, no. 11, pp. 2061-2069, Nov. 2006.

[10] B. Fan, Wen. Wang, Y. Lin, L. Huang, K. Zheng, "Subcarrier Allocation for OFDMA Relay Networks with Proportional Fair Constraint," in Proc. IEEE Conf. on ICC, pp. 1-5, 2009.

[11] J. Yuan, Q. Wang, "Adaptive Resource Allocation Schemes for Multiuser OFDMA Nonregenerative Relay Networks," in Proc. IEEE Conf. on ICC, pp. 1-5, 2010.

[12] M. Hajiaghayi, M. Dong, and B. Liang, "Optimal channel assignment and power allocation for dual-hop multi-channel multi-user relaying," in Proc. IEEE Conf. on INFOCOM, Mini-Conference, pp. 76-80, Apr. 2011.

[13] V. Luc, L. Jerome, O. Onur, and Z. Abdellatif, "Rate-Optimized Power Allocation for DF-Relayed OFDM Transmission under Sum and Individual Power Constraints", EURASIP Journal on wireless comm. and networking, Vol. 2009, 2009.

[14] H. Boostanimehr, V. K. Bhargava, "Selective Subcarrier Pairing and Power Allocation for DF OFDM Relay Systems with Perfect and Partial CSI," IEEE Trans. Wireless Commun., vol. 10, no. 12, pp. 4057-4067, Dec. 2011.

[15] W. Yu and R. Lui, "Dual methods for nonconvex spectrum optimization of multi-carrier systems," IEEE Trans. Commun., vol. 54, no. 7, pp. 1310-1322, Jul. 2006.

[16] H. W. Kuhn, "The hungarian method for the assignment problem," Naval Research Logistics, vol. 52, pp. 7-21, 2005. 